\title{
CLIMA DE AULA Y BUENAS PRÁCTICAS DOCENTES CON ADOLESCENTES VULNERABLES: MÁS ALLÁ DE LOS CONTENIDOS ACADÉMICOS
}

\author{
Marco Antonio Manota Sánchez \\ Escuela Virgen de Guadalupe (Badajoz) \\ Miguel Melendro Estefanía \\ Universidad Nacional de Educación a Distancia (UNED)
}

\begin{abstract}
RESUMEN: El objetivo del presente artículo es analizar una serie de elementos relacionados con las buenas prácticas docentes, que inciden de forma importante en la configuración del clima de aula, especialmente con alumnado vulnerable. Se parte de las percepciones de profesores-tutores y alumnos del primer ciclo de la ESO contrastadas a partir de tres instrumentos: el Test de Adaptación Multifactorial Infantil (TAMAI) y dos cuestionarios sobre "Percepción del Clima de Aula por el Alumnado" (PCA-A) y "Percepción del Clima de Aula por el Profesorado" (PCA-P).

Entre los resultados obtenidos, como buenas prácticas docentes en relación al clima de aula se destacan la actitud de escucha del profesorado hacia los alumnos/as, su disponibilidad para acompañarles en el proceso educativo y neutralizar la creación de estereotipos y etiquetas excluyentes, la importancia de la formación del profesorado en la gestión de las emociones y, sobre todo, de su predisposición para compartir esas emociones. Otros resultados significativos muestran la desigual necesidad de escucha de los alumnos, el "punto ciego" que suponen las preconcepciones negativas mutuas entre profesorado y alumnado y la forma en que las percepciones de los alumnos cambian dependiendo del grado de vulnerabilidad y el nivel de inadaptación escolar que presentan.
\end{abstract}

PALABRAS CLAVE: Relación profesor-alumno, buena práctica, clima de aula, alumno vulnerable, competencia, inadaptación.

\section{CLASSROOM'S CLIMATE AND GOOD TEACHING METHODS WITH VULNERABLE TEENAGERS: FURTHER FROM ACADEMIC CONTENTS}

\footnotetext{
ABSTRACT: The aim of this article is to analyse a series of elements related with good teaching practices, which are of remarkable importance in
} 
the configuration of the classroom climate, especially regarding vulnerable students. We start from the perception of teachers, tutors and students from first cycle of secondary education contrasted with three instruments: the "Child Multifactorial Adaptation Test" (TAMAI) and two surveys about "Perception of the classroom climate by the students" (PCA-A), and "Perception of the classroom climate by the teachers" (PCA-P).

Between obtained results, as good teaching practices in relation with the climate of the classroom, it is pointed out the listening attitude from the teacher towards the alumni, their availability to accompany them on their educative process and neutralise the creation of stereotypes and excluding labels, the importance of the formation of the teachers in the emotions management, and above all, their inclination to share those emotions. Other significant results show the uneven necessity of being heard of the students, the "blind spot" of negative misconceptions between teachers and students, and the way that students' perception change depending on their vulnerability degree and the level of failure at adapting to school they might present.

KEYWORDS: Teacher student relationship, best practice, classroom environment, disadvantaged student, competence, maladjustment.

Recibido: 24/04/2015

Aceptado: 06/06/2015

Correspondencia: Marco Antonio Manota Sánchez, Escuela Virgen de Guadalupe, C/ Corte de Peleas, 79, 06009 Badajoz. Email: manotasanchez@gmail.com.

\section{INTRODUCCIÓN}

Un importante factor de buenas prácticas en escuelas eficaces es la existencia de un buen clima escolar, así como experiencia y formación en convivencia y resolución de conflictos por parte de los centros educativos (Lizasoain y Angulo, 2014). Tanto es así que se puede considerar con Amores y Ritacco (2012) que la labor relacional debe preceder a la enseñanza de conocimientos, con el objetivo de dar lugar a la creación de vínculos personales como elemento de mejora del clima de aula.

El clima escolar se encuentra mediatizado por un contexto configurado a su vez por múltiples factores en diferentes niveles: organizativo o institucional, de aula e intrapersonal. Así, en el aula se dan cita tanto elementos humanos, como la interacción entre alumnado y profesorado dentro de un contexto socioeducativo definido, como otros procesos más relacionados con los contenidos objeto de enseñanza (Hernández y Hernández, 2011; Cornejo y Redondo, 2001). El clima de aula se construye a partir de las normas, hábitos, ritos, comportamientos y prácticas sociales que emergen de las relaciones que profesores y alumnos establecen entre sí, formando unas pautas socioafectivas determinadas. Y también a partir de la percepción del alumno sobre su acogida por los demás, su pertenencia a la institución, la relación con el profesor y con sus compañeros. Esta percepción del alumno puede coincidir o no 
con la percepción del profesor, pero se enmarca en una dinámica de intercambio de interpretaciones que es parte esencial de la naturaleza del proceso educativo (Claro, 2013; García Amilburu, 2002).

Por otro lado, la responsabilidad de que el grupo aprenda a relacionarse recae sobre la persona adulta, o lo que es lo mismo, sobre el profesor. En este sentido, los profesores eficaces en la mejora de las habilidades sociales y del comportamiento del alumnado, proporcionarán un impulso fundamental a la adquisición de competencias académicas y la enseñanza de contenidos académicos (Comellas, 2013; Boyano, 2013; Bueno y Garrido, 2012). Efectivamente, se ha comprobado que la percepción de las relaciones interpersonales influye tanto en el clima de aula -que a su vez se genera a partir de las interpretaciones que los grupos hacen de él-como en el logro de objetivos académicos (Moreno, Díaz, Cuevas, Nova y Bravo, 2011; Murillo y Becerra, 2009).

En este sentido, los profesores-tutores, tomados como referencia en la presente investigación, cumplen una función muy importante para el desarrollo integral de los alumnos a través de relaciones interpersonales de calidad. De alguna manera, ser tutor "significa siempre protección y cuidado, orientación y guía" (López Gómez, 2013); de ahí que se plantee que los problemas de indisciplina dentro de una clase pueden predecirse a través del nivel de competencia afectiva interpersonal del profesor (Moreno et al., 2011). La conducta del alumno no dependería por tanto exclusivamente de la materia de estudio, sino que variaría en función de su percepción del clima de aula en situaciones concretas, y su éxito académico no dependería sólo de él, sino de otros factores, entre ellos y de forma relevante de las actitudes y comportamientos de sus profesores (Carbonero, Martín, Román y Reoyo, 2010).Coincidimos con Pilar Arnaiz (2011) cuando afirma, refiriéndose a la educación inclusiva, que si se quiere pensar en la heterogeneidad del alumnado como una situación normal, se debe poner en marcha una planificación educativa que permita utilizar a los profesores recursos instrumentales y actitudinales, pero también recursos intrapersonales e interpersonales en beneficio de todos los alumnos.

A todo ello habría que sumarle la función socioeducativa de la escuela, escasamente explicitada pero que posee gran importancia. Como plantea González Alemán (2010), esta función puede entenderse como un proceso de "relación", en el que se trabaja desde una "apuesta por las personas" y su protagonismo, y de "la transformación de la sociedad en clave de valores" con el objetivo principal de proporcionar a los niños y jóvenes una "formación plena" que les permita conformar su "propia y esencial identidad". Siguiendo esta reflexión, el profesor se convierte en un representante del mundo con la responsabilidad de ser el otro que necesita el alumno, y que articula referentes culturales amplios (Fryd, 2011) o, dicho de otro modo, que "tiende puentes" educando (García Amilburu, 2002). En este sentido, en investigaciones realizadas tanto en escuelas eficaces como en torno a prácticas escolares ante la exclusión social, se detectó que en ambos casos los profesores se implican especialmente en la tarea educativa o formativa, más allá de la meramente instructiva (Lizasoain y Angulo, 2014; Amores y Ritacco, 2012). De esta manera, la evaluación de alumnos en riesgo de fracaso y exclusión socioeducativa se asocia con una tendencia pedagógica que da más importancia al papel de la enseñanza en el desarrollo personal y social, el aprendizaje individual y grupal, el carácter comunicativo de la educación y las relaciones menos directivas y autoritarias entre profesores y alumnos (Amores y Ritacco, 2011). 
Se constata que los retos planteados al profesorado en este sentido son amplios y complejos:

Se le pide una relación personal con el alumno, un trabajo en equipo, y a la vez que promueva la calidad, la excelencia, la evaluación individualizada y competitiva. Se pretende un profesor agente compensador de las desigualdades sociales y de las deficiencias personales (...) y al mismo tiempo se le demanda énfasis en los aprendizajes de contenidos disciplinares (García García, 2010: 34-35).

En la realidad se destaca, sin embargo, cómo el desconocimiento de las raíces y los procesos de transformación de los problemas, la actuación por intuición y la realización de prácticas acostumbradas, así como la defensa-reacción ante lo que se percibe como agresión, se encuentran entre las causas que llevan a los profesores a simplificar los procesos de actuación pedagógica (Ochoa y Peiró, 2010) y a dar respuestas simples a problemas complejos de ámbito social, cultural e interpersonal. Son los factores psicosociales, contextualizados en la interacción social, los que explican el fracaso escolar, sin embargo los profesores tienden a infravalorarlos y a poner demasiado énfasis en variables personales (Bueno y Garrido, 2012) y más concretamente en las aspectos personales del alumno que fracasa.

Como se señaló anteriormente, el clima escolar se encuentra condicionado por los contextos de pertenencia de los sujetos que forman parte de la comunidad educativa (Claro, 2013). En relación a la población más vulnerable, aunque resulte difícil contrarrestar los factores de riesgo socioeconómico o familiar de los alumnos, sí es posible abrir espacios relacionales que ayuden a construir y/o afianzar sus identidades, rompiendo la cadena que les mantiene atrapados en su situación de partida. El sentido de pertenencia al grupo que se logra con estos procesos resulta fundamental para la prevención de la violencia y para el éxito educativo (Comellas, 2013), así como para ofrecer a los alumnos más vulnerables la oportunidad de compensar las dificultades sociales existentes y aproximarles a una realidad más inclusiva.

\section{Metodología}

En la realización de este trabajo se utilizó una metodología de estudio de casos de tipo descriptivo y cuantitativo, a partir de una población de profesores-tutores y alumnos del Primer Ciclo de la ESO en tres centros educativos de características similares ${ }^{1}$, en tres comunidades autónomas diferentes: Extremadura (Badajoz), Castilla y León (Valladolid) y Andalucía (Huelva).

Estos tres centros fueron seleccionados para el estudio de casos debido a que ofrecen una oferta formativa similar a una población heterogénea, desde Bachillerato y Formación Profesional de Grado Medio a Formación Profesional Básica y antiguos Programas de Cualificación Profesional Inicial, destinados a alumnos que abandonan la enseñanza obligatoria sin superar $2^{\circ}$ ESO y que desean formarse para entrar en el

1. Escuela Virgen de Guadalupe, Instituto Politécnico Cristo Rey y Escuelas Profesionales Safa Funcadia. Los tres centros ofrecen una propuesta que va desde Educación Infantil hasta Bachillerato y/o Ciclos Formativos (figura 1); su identidad y su estilo educativo son responsabilidad última de la entidad que los gestiona, la Compañía de Jesús. 
mundo del trabajo. Cada oferta formativa responde a las necesidades de perfiles muy distintos de alumnos, lo que da una idea de la heterogeneidad de los distintos entornos socio-familiares de procedencia y de los intereses del alumnado de los tres centros.

Por otro lado, dichos centros pertenecen a la misma organización -Compañía de Jesús-, con lo que las directrices y planteamientos educativos tienen una misma base y suponen prácticas educativas muy similares.

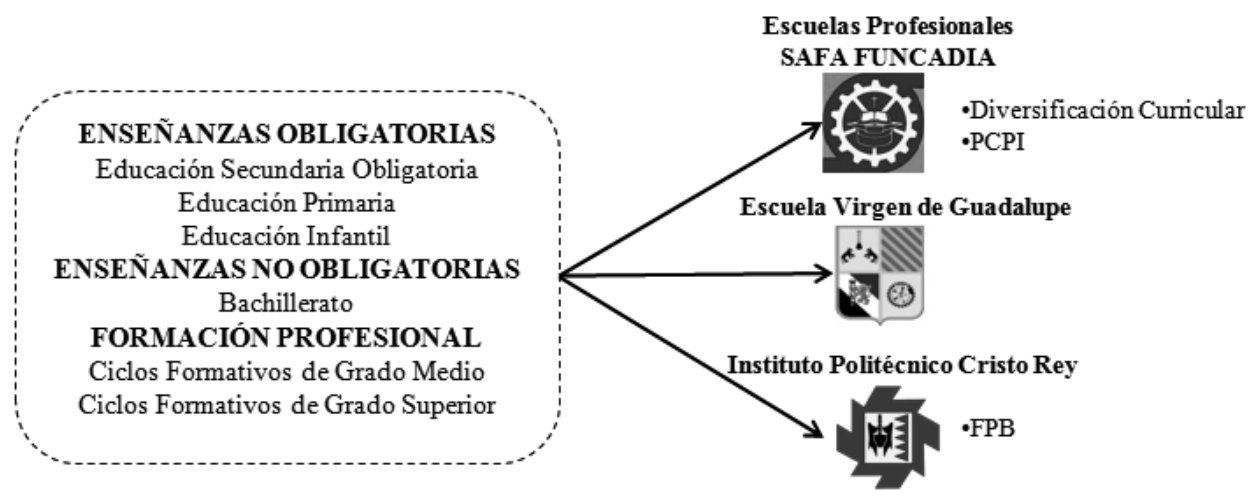

Figura 1. Oferta educativa de los centros seleccionados

El estudio de casos es abordable desde la utilización de metodologías cuantitativas (Simons, 2011), opción seguida en el presente estudio, en el que se administró una prueba estandarizada a alumnos, el Test Autoevaluativo Multifactorial de Adaptación Infantil (TAMAI)(Hernández-Guanir, 2009), y se diseñaron y aplicaron dos cuestionarios ad hoc, relacionados entre sí de forma directa: un cuestionario sobre "Percepción del Clima de Aula por el Alumnado" (PCA-A) y otro sobre "Percepción del Clima de Aula por el Profesorado" (PCA-P)

EI TAMAI se utilizó para identificar a los alumnos con inadaptación escolar, descrita a partir de dos variables: la aversión a la instrucción y la indisciplina. La primera se define como la disposición negativa hacia las materias de aprendizaje y el profesorado, y contiene a su vez tres ítems: hipolaboriosidad, que se define como el bajo esfuerzo, rendimiento, aplicación y rendimiento académico, así como la distracción; hipomotivación, que se caracteriza por el bajo interés hacia los contenidos académicos, el aburrimiento y la insatisfacción hacia la institución escolar y hacia alguna materia en particular; y aversión al profesor, que se caracteriza por la actitud negativa hacia el profesor y su modo de enseñar. Por otro lado, la indisciplina se caracteriza por el comportamiento disruptivo e inadecuado.

Los cuestionarios ad hoc (PCA-A y PCA-P) fueron diseñados a partir de cuatro dimensiones centrales de la presente investigación (figura 2): relación interpersonal, percepción del clima de aula, intervención del profesorado y educación integral. La relación interpersonal analiza la percepción que tienen los profesores sobre su gestión emocional y la distancia entre los roles asignados por la institución y la relación genuina entre personas. La percepción del clima de aula contrasta las percepciones sobre el ambiente en la clase por parte de profesores y alumnos, y la 
influencia que sobre ellas tienen los estereotipos mutuos. La dimensión sobre intervención del profesorado se centra en el análisis de los recursos del profesorado para mejorar la relación de ayuda con los alumnos en el ámbito escolar, así como la convivencia en el aula. Finalmente, en relación a la dimensión sobre educación integral, se aborda la relación entre los planteamientos de una educación integral, desde una perspectiva socioeducativa, y una educación centrada en los contenidos académicos.

\begin{tabular}{|c|c|}
\hline \multicolumn{2}{|c|}{ Dimensión 1: RELACIÓN INTERPERSONAL } \\
\hline FAMILIAS & CATEGORÍAS \\
\hline D1A. Recursos del profesorado & $\begin{array}{ll}\text { Formación sobre: } \\
\text { - } \quad \text { Inteligencia Emocional } \\
\text { - } \quad \text { Cuidado de la relación interpersonal } \\
\text { - } \quad \text { Clima de aula } \\
\end{array}$ \\
\hline D1B. Roles & $\begin{array}{l}\text { - } \quad \text { Roles asignados por la institución. } \\
\text { - } \quad \text { Obligatoriedad de la asistencia al centro escolar } \\
\text { - Asimetría en la interacción profesor-alumno }\end{array}$ \\
\hline D1C. Conflictos & $\begin{array}{ll}\text { - } & \text { Prevención de conflictos } \\
\text { - } & \text { Resolución de conflictos } \\
\end{array}$ \\
\hline \multicolumn{2}{|c|}{ Dimensión 2: PERCEPCIÓN DEL CLIMA DE AULA } \\
\hline FAMILIAS & CATEGORÍAS \\
\hline D2A. Condicionantes & $\begin{array}{ll}\text { - } & \text { Percepciones mutuas entre profesores y alumnos } \\
\text { - } & \text { Ideas preconcebidas entre profesores y alumnos }\end{array}$ \\
\hline \multicolumn{2}{|c|}{ Dimensión 3: INTERVENCIÓN DEL PROFESORADO } \\
\hline FAMILIAS & CATEGORÍAS \\
\hline D3A. Relación de ayuda & $\begin{array}{ll}\text { - } & \text { Empatía } \\
\text { - } & \text { Escucha activa } \\
\text { Aceptación incondicional }\end{array}$ \\
\hline D3B. Convivencia en el aula & - $\quad$ Clima de aula \\
\hline \multicolumn{2}{|c|}{ Dimensión 4: EDUCACIÓN INTEGRAL } \\
\hline FAMILIAS & CATEGORÍAS \\
\hline D4A. Contenidos & $\begin{array}{l}\text { - } \quad \text { Tipos de contenidos transmitidos } \\
\text { - } \quad \text { Evaluación de los objetivos expresados }\end{array}$ \\
\hline $\begin{array}{l}\text { D4B. Intervención } \\
\text { socioeducativa }\end{array}$ & $\begin{array}{ll}\text { - } & \text { Transmisión de valores } \\
\text { - Formación. }\end{array}$ \\
\hline
\end{tabular}

Figura 2. Dimensiones de investigación

El Cuestionario PCA-P (profesorado) consta de 39 preguntas cerradas y el Cuestionario PCA-A (alumnado) consta de 35 preguntas también cerradas. Ambos utilizan una Escala Likert 1-4 (muy en desacuerdo, en desacuerdo, de acuerdo y muy de acuerdo).

Las preguntas elaboradas ayudan a describir la percepción de profesores-tutores y alumnos, buscando minimizar los efectos de la indefinición, la deseabilidad social o la excesiva abstracción (Alvira, 2011). Se incluyeron así mismo preguntas de control para poder evaluar la fiabilidad de la prueba. Se realizó la validación previa 
de los dos cuestionarios por parte de cinco expertos procedentes tanto del ámbito académico como del profesional ${ }^{2}$ (Alvira, 2011), y una prueba piloto con ocho alumnos y cuatro profesores-tutores.

Las pruebas fueron aplicadas de forma individualizada a una muestra intencional -a partir de los centros seleccionados para el estudio de casos- de alumnos y profesores-tutores del Primer Ciclo de la ESO. En cuanto al alumnado, de una población de 540 alumnos, 263 cumplimentaron el TAMAI y el PCA-A (alumnado). Los alumnos de la muestra presentaban una media de edad de 12,86 años, y una moda de 13 años; el $51 \%$ se encontraba en $1^{\circ}$ de ESO y el otro $49 \%$, en $2^{\circ}$ de ESO; por otro lado, el $55 \%$ eran varones y el $45 \%$ mujeres. Del total de la muestra, el $49 \%$ (128) presentaba inadaptación escolar en distinto grado y el 51\% (135) no la presentaban.

En cuanto a los profesores-tutores, participaron un número total de 13, con una edad media de 45 años, de los cuales 5 eran hombres y 8 mujeres. Así mismo, 7 eran tutores de $1^{\circ} \mathrm{ESO}$ y 6 de $2^{\circ} \mathrm{ESO}$.

\section{Resultados Y DISCUSIÓN}

A continuación se destacan los principales resultados del estudio, así como los elementos para la reflexión que se aportan desde otras investigaciones similares. En el proceso de triangulación, se seleccionaron aquellas respuestas de profesores y alumnos que muestran contrastes o coincidencias significativas e interesantes en relación al tema investigado.

Como se señalaba, el TAMAI resultó útil inicialmente para establecer la delimitación entre población inadaptada escolarmente -en diferentes grados-y aquella que no presentaba ningún grado de inadaptación escolar. Así se establecieron cuatro niveles de inadaptación escolar ${ }^{3}$ a partir de los datos que nos ofrecía el test: un primer nivel, que se denominó "sin IE", en el que no se constata la existencia de inadaptación escolar (centil 1 al 60); un segundo nivel, denominado "IE baja", en el que se constata inadaptación escolar (centil61 al 80); un tercer nivel, denominado "IE media", en el ésta se encuentra bien constatada (centil 81 al 95); y un cuarto criterio, denominado "IE alta", en el que la inadaptación escolar se encuentra muy constatada (centil 96 al 99).

Por otra parte, con el objetivo de analizar la posible relación entre la Inadaptación Escolar y la Inadaptación Social, con los cuatro niveles de Inadaptación Social se realizó el mismo proceso, identificando tres niveles de IS: "sin IS", "IS baja", "IS media" e "IS alta". Se comprobó que en el grupo de alumnos con algún grado de inadaptación escolar (IE) el 71\% presentaba también inadaptación social (IS); y que, por otro lado, en el grupo de alumnos sin inadaptación escolar (IE), el 80\% tampoco presentaba Inadaptación Social (IS).

2. Cinco expertos con amplia formación y experiencia, dos pertenecen al ámbito universitario y con una experiencia de 20 años de investigación en los siguientes campos: ciencias sociales y humanidades, formación del profesorado, métodos de investigación en educación, asesoramiento en intervención psicopedagógica, investigación socioeducativa y educación en valores e investigación en calidad de la enseñanza y tres expertos en el ámbito profesional con una experiencia de 17 años en los siguientes campos: educación de calle con menores en riesgo e infractores, orientación laboral de personas en exclusión, acompañamiento a alumnos con dificultades sociofamiliares y discapacidad.

3. A partir de aquí, denominaremos IE a la inadaptación escolar e IS a la inadaptación social. 
Por último, el valor de la correlación de Pearson entre ambas inadaptaciones fue de $r=0,38$, con una significación de $p \leq 0,01$. Estos resultados indican que existe una fuerte relación entre ambas inadaptaciones, aunque no se puede generalizar la idea de que los alumnos con Inadaptación Social presentan siempre inadaptación escolar; cuestiones ambas que debemos tener en cuenta a la hora de intervenir con los alumnos.

\section{Sin ningún grado de IE Con algún grado de IE}
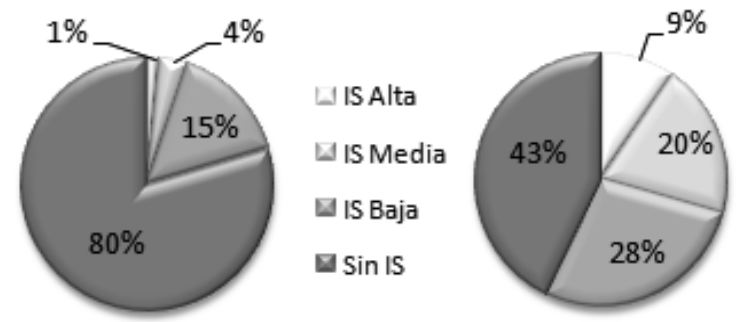

\begin{tabular}{|c|c|c|c|}
\hline & & $\begin{array}{l}\text { Inadaptación } \\
\text { Escolar }\end{array}$ & $\begin{array}{l}\text { Inadaptación } \\
\text { Social }\end{array}$ \\
\hline $\begin{array}{l}\text { Inadaptación } \\
\text { Escolar }\end{array}$ & $\begin{array}{l}\text { Correlación de } \\
\text { Pearson } \\
\text { Sign. (2-colas) } \\
N\end{array}$ & 1.00 & $\begin{array}{r}.38 \\
.000 \\
263\end{array}$ \\
\hline $\begin{array}{l}\text { Inadaptación } \\
\text { Social }\end{array}$ & $\begin{array}{l}\text { Correlación de } \\
\text { Pearson } \\
\text { Sign. (2-colas) } \\
N\end{array}$ & $\begin{array}{r}.38 \\
.000 \\
263\end{array}$ & $\begin{array}{l}1.00 \\
263\end{array}$ \\
\hline
\end{tabular}

Figura 3. Relación entre la IE y la IS

Como se puede observar en la figura 4, los datos más significativos se corresponden con los alumnos de 12 y 15 años. El porcentaje de alumnos con 12 años desciende en la medida en que la IE aumenta. Sin embargo, el tanto por ciento de alumnos con 15 años se concentra de manera muy significativa en la IE Alta.

En cuanto a los niveles educativos, en la figura 5 se aprecia la incidencia de la $\mathrm{IE}$, que se incrementa en relación al curso: aumenta en referencia a $2^{\circ} \mathrm{ESO}$ y disminuye en referencia a $1^{\circ} \mathrm{ESO}$, lo que indica que ésta tiende a agravarse a medida que el curso es de nivel más avanzado. Por otra parte, y a partir de lo que este dato indica, se observa que con la repetición de curso tiende a incrementarse la inadaptación escolar; lo que lleva a preguntarse por la conveniencia de que los alumnos de 15 años promocionen a $3^{\circ}$ ESO, y las condiciones en que esa promoción se realiza. En este sentido, inicialmente y con la información disponible, la repetición parece no sólo agravar la situación de inadaptación escolar de los alumnos sino que se puede considerar un predictor de abandono escolar (Mena, Fernández y Riviere, 2010).

A partir de aquí se triangularon los resultados del Cuestionario a profesores y del Cuestionario a alumnos, en relación a los diferentes objetivos de la investigación y con especial atención al grado de IE de los alumnos. 

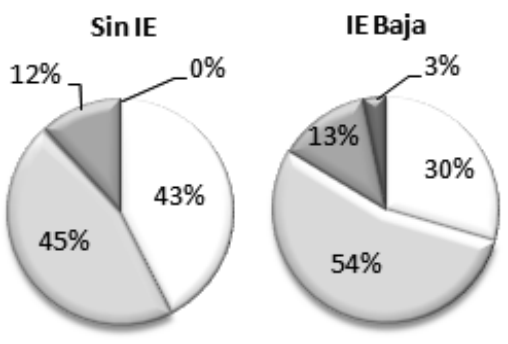

IE Media

IE Alta
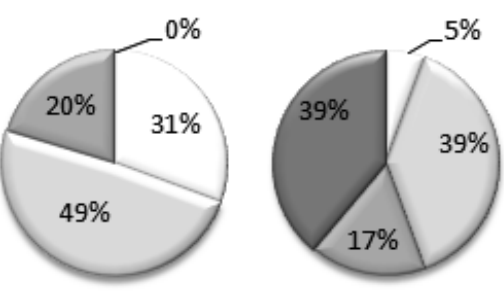

$\sqcup 12$ años

$\triangle 13$ años

$\square 14$ años

$\square 15$ años

\begin{tabular}{||l|l|l|r||}
\hline \multicolumn{2}{||l|}{} & $\begin{array}{l}\text { Inadaptación } \\
\text { Escolar }\end{array}$ & Edad \\
\hline $\begin{array}{l}\text { Inadaptación } \\
\text { Escolar }\end{array}$ & $\begin{array}{l}\text { Correlación de } \\
\text { Pearson } \\
\text { Sign. (2-colas) } \\
N\end{array}$ & 1.00 & .31 \\
& $\begin{array}{l}\text { Correlación de } \\
\text { Eearson } \\
\text { Edad }\end{array}$ & 263 & .000 \\
& $N$ & .31 & 1.00 \\
\hline
\end{tabular}

Figura 4. Inadaptación Escolar por edades

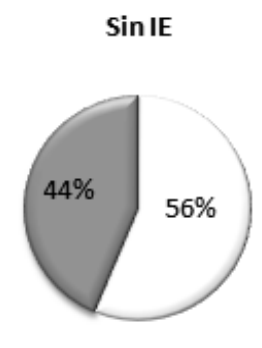

IE Baja

IE Media

IEAlta
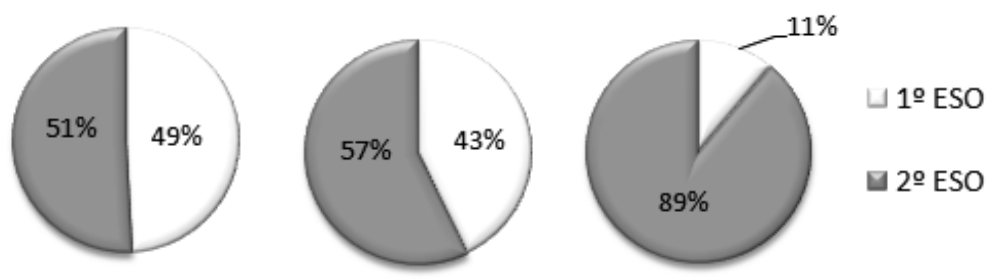

\begin{tabular}{lcccc} 
& Sin IE & IE Baja & IE Media & IE Alta \\
\cline { 2 - 5 } $\mathbf{1}^{\mathbf{0}}$ ESO & $56 \%$ & $49 \%$ & $43 \%$ & $11 \%$ \\
\cline { 2 - 5 } $\mathbf{2}^{\mathbf{0}}$ ESO & $44 \%$ & $51 \%$ & $57 \%$ & $89 \%$
\end{tabular}

\begin{tabular}{|c|c|c|c|}
\hline & & $\begin{array}{l}\text { Inadaptación } \\
\text { Escolar }\end{array}$ & Curso \\
\hline $\begin{array}{l}\text { Inadaptación } \\
\text { Escolar }\end{array}$ & $\begin{array}{l}\text { Correlación de } \\
\text { Pearson } \\
\text { Sign. (2-colas) } \\
N\end{array}$ & $\begin{array}{l}1.00 \\
263\end{array}$ & $\begin{array}{l}.21 \\
.001 \\
263\end{array}$ \\
\hline Curso & $\begin{array}{l}\text { Correlación de } \\
\text { Pearson } \\
\text { Sign. (2-colas) } \\
N\end{array}$ & $\begin{array}{r}.21 \\
.001 \\
263\end{array}$ & 1.00 \\
\hline
\end{tabular}

Figura 5. Inadaptación Escolar por cursos

Un primer resultado destacable desde la triangulación muestra la relación entre la actitud de escucha del profesorado y la percepción de los alumnos sobre ella (figu- 
ra 6). Así, mientras que para prácticamente la totalidad del profesorado encuestado $(85 \%)$ la escucha es una de sus principales labores docentes, no todos los grupos de alumnos sienten confianza para hablar de temas personales con sus profesores. La percepción de los alumnos sobre este tema es proporcionalmente divergente en función de su grado de inadaptación escolar: mientras que los alumnos que no presentan IE sienten confianza para hablar de temas personales con sus profesores (61\%), esta percepción disminuye progresivamente entre los que presentan un grado de IE bajo o medio (58\% y $42 \%$ respectivamente), para disminuir de forma muy significativa en aquellos alumnos con un grado de IE alto (22\%). Se observa que la escucha activa como buena práctica por parte del profesorado debe responder a las diferentes necesidades de los alumnos, además de saber acompañarles con el objetivo de suavizar las resistencias a hablar con el profesor. Como señalan Melendro, González y Rodríguez (2013: 116), algunas de las principales cualidades y habilidades personales del educador tienen que ver con su capacidad para "la escucha activa, la sinceridad y transparencia, las habilidades de comunicación, el manejo del humor y el uso adaptado del lenguaje con los adolescentes y, como síntesis de todas ellas, la disposición para abordar el cambio personal".

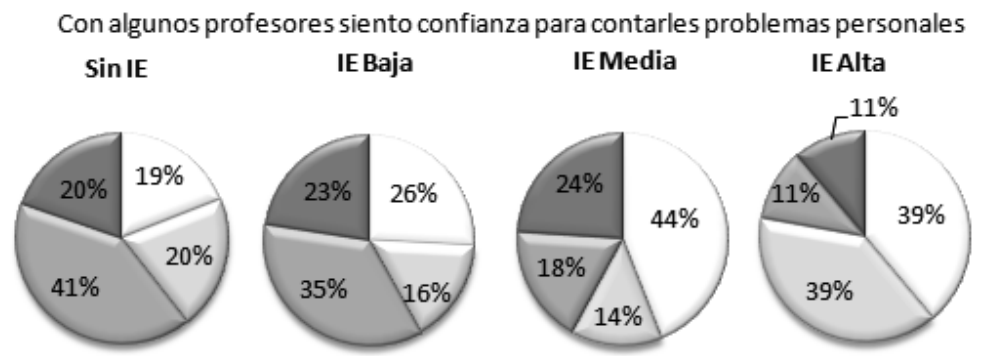

Una de las funciones principales de mi labor docente, además de la académica, es escuchar a mis alumnos Tutores

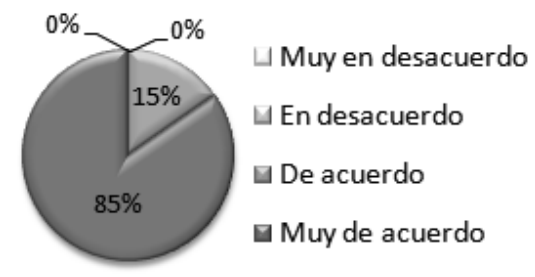

Figura 6. Labor de escucha del profesor

Los resultados que devuelve la figura 7 concuerdan con lo que plantea Arellano (2006), acerca de la necesidad de que los profesores vayan más allá del interés por establecer la escucha, y posean los recursos necesarios para identificar si en el proceso de comunicación con los alumnos existen barreras que impiden una comunicación asertiva y efectiva profesor-alumno.

En este sentido se manifiestan también Moreno et al. (2011) cuando muestran la relación significativa que existe entre percepción del clima escolar y desarrollo 
emocional de alumnos y profesores. Así, se constata en el presente estudio que cuando los alumnos perciben un estado emocional negativo (enfado) del profesorado, sienten que esto influye negativamente en el clima de aula. Esta percepción aumenta en la medida en la que la desadaptación del alumno es mayor: en los alumnos sin IE Ilega al 89\%, mientras que en los alumnos con IE media o baja se sitúa entre el $92 \%$ y el $94 \%$, y entre los que presentan una IE alta supone el $100 \%$ de las respuestas. Sin embargo se observa que el $85 \%$ los profesores-tutores no cree que en esas situaciones, por ejemplo hablar en clase sobre su estado de ánimo, ayude a mejorar el clima de aula. Una adecuada gestión emocional por parte del profesorado facilita el uso educativo de la comunicación de su estado de ánimo a los alumnos, lo que supondría una buena práctica, ya que favorece que los alumnos interpreten adecuadamente la actitud del profesor y aprendan a gestionar sus propias emociones.

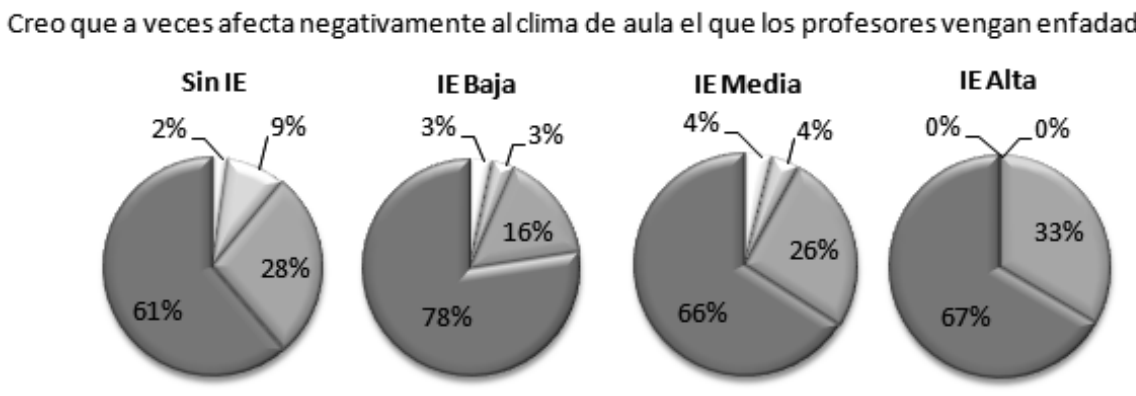

Hablar sobre mi estado de ánimo al comenzar la clase no ayuda en nada al clima de aula

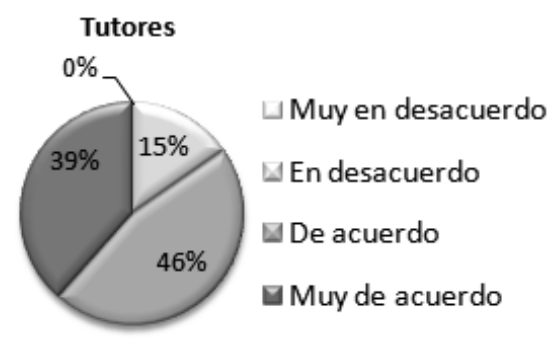

Figura 7. Emociones del profesor y clima de aula

La docencia se desarrolla dentro de un contexto influido, entre otras cosas, por las percepciones subjetivas y por las características de alumnos y profesores (Zárate, 2002), de forma que la valoración mutua entre tutor y alumnos influye de forma significativa en los resultados académicos de estos últimos (Lozano, 2003). La figura 8 muestra que mientras los alumnos afirman no sentirse afectados por las opiniones de sus compañeros en su relación con los profesores: alumnos sin IE (77\%), con IE baja $(74 \%)$, media (68\%) y alta (73\%); los profesores-tutores afirman $(61 \%)$ que conocer la "mala fama" de un alumno/a no les condiciona en su relación con él/ella. Es decir, la mayoría afirma que las informaciones previas de terceros, y en este caso negativas, 
sobre los alumnos no les generan un estereotipo y, por tanto, no les afectan en su relación con ellos. Sin embargo, las respuestas de los profesores-tutores no son concluyentes al responder si las preconcepciones sobre algunos alumnos no les afectan en su relación con ellos, repartiéndose éstas entre las opciones "de acuerdo" (54\%) y "en desacuerdo" (46\%).

Ante esta ambigüedad, parece necesario que los profesores se conciencien de la existencia de los estereotipos y de que en su mano está controlar que no les afecten en el aula, ya que como comenta Furrer (2013) relacionarse desde esa perspectiva con los alumnos afectará, entre otras cuestiones, a la percepción que éstos tienen de sí mismos. En este sentido, las buenas prácticas docentes pasan necesariamente por el cuidado, por parte de los profesores, al hablar entre ellos de alumnos con dificultades de aprendizaje y, especialmente, con dificultades para seguir las normas o para relacionarse con los demás. En este sentido, se debe evitar la creación y potenciación de estereotipos.

Por otro lado, y en congruencia con lo dicho anteriormente, en la figura 9 se puede comprobar que en la medida en que aumenta la IE de los alumnos, también aumenta su sensación de cargar con el estereotipo de "problemáticos" asignado por los profesores. Por otro lado, esa misma relación se aprecia entre la IE y el malestar de los alumnos en el aula que, según su percepción, también es debido a los profesores. Estos resultados sugieren que la IE mantiene relación con la percepción de cargar con un estereotipo y con el malestar en el aula. De esta manera, sus respuestas devuelven una correlación de Pearson de $r=-0,27$ y una significación de $p \leq 0,01$.

Lo que me dicen los demás alumnos sobre los profesores no me afecta en mi relación con ellos

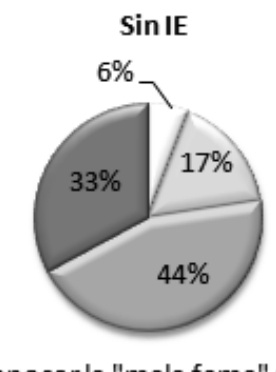

IE Baja

IEMedia

IEAlta
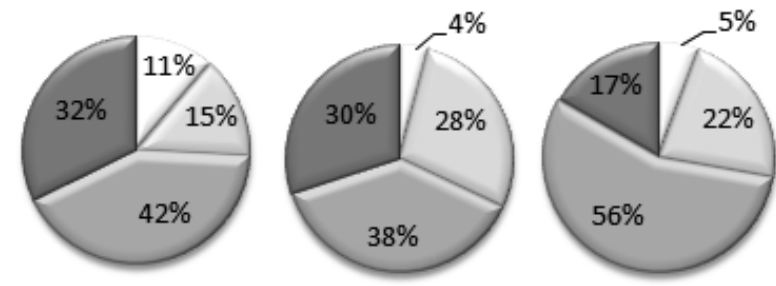

Conocer la "mala fama" de un alumno me ayuda a prever los problemas que voy a tener con él

Las preconcepciones sobre algunos alumnos no me influyen en mi relación con ellos.

Tutores

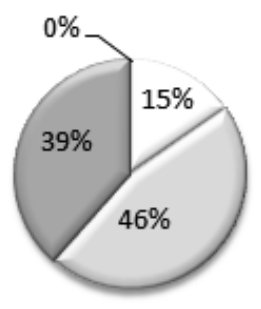

$\sqcup$ Muy en desacuerdo

$\square$ En desacuerdo

$\square$ De acuerdo

Muy de acuerdo

\section{Tutores}

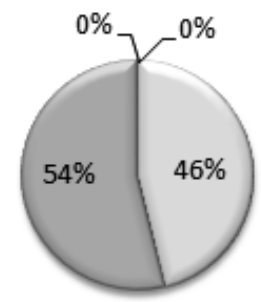

Figura 8. Preconcepciones mutuas de profesores y alumnos 
Los profesores me miran mal porque tengo fama de provocar problemas
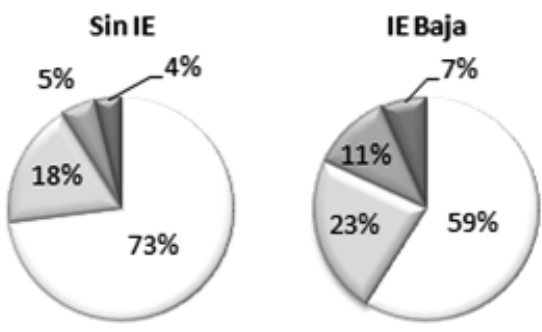

IEMedia

IEAlta
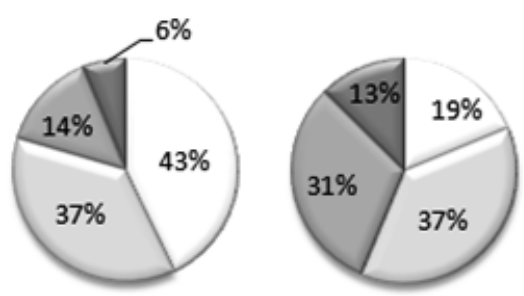

$\sqcup$ Muy en desacuerdo

$\square$ En desacuerdo

$\square$ De acuerdo

Muy de acuerdo

Todos los profesores consiguen que me sienta bien en el aula

Sin IE

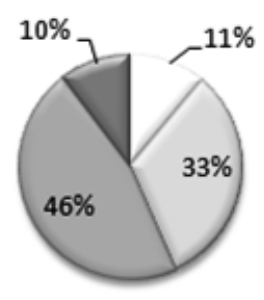

IE Baja

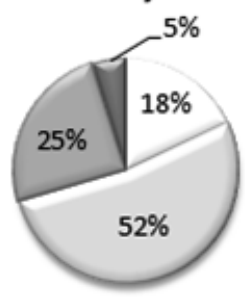

IEMedia

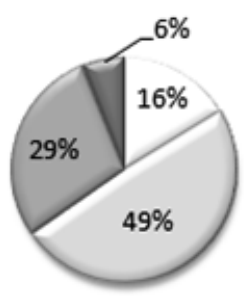

IEAlta

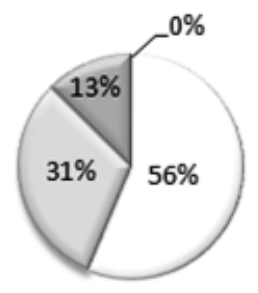

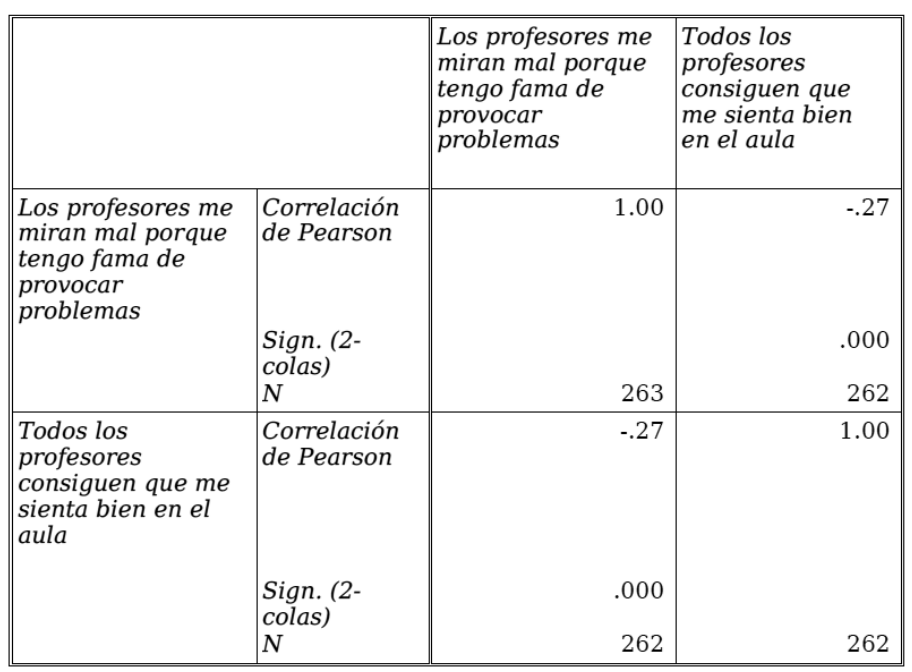

Figura 9. Interpretación de la percepción negativa de los profesores y su correlación negativa con la percepción sobre éstos como factor de bienestar 
Por su parte los alumnos consideran que los profesores les ayudan a sentirse bien consigo mismos y con los demás. Así lo afirma el $81 \%$ de los alumnos sin IE, un 57\% de los alumnos con IE baja, el $50 \%$ de los que presentan una IE media y el $47 \%$ de los que presentan IE alta. Es interesante comprobar, en base a estos datos, como cuando aumenta el grado de inadaptación escolar disminuye la percepción positiva de la ayuda que puede brindar el profesorado.

En congruencia con investigaciones en las que se ha mostrado que las expectativas del profesor influyen en la ejecución académica de los alumnos (Jiménez, 2013; López Gómez, 2013), éstos, efectivamente, se perciben con más fuerza para aprobar si un profesor cree en ellos (figura 10). De la misma manera, la totalidad del profesorado encuestado afirma que creer en las posibilidades de un alumno afecta positivamente a la percepción que el alumno tiene de sí mismo. Cabe destacar, sin embargo, que esta percepción sobre su capacidad para aprobar es mayor en los alumnos sin IE (93\%), y que en la medida en que la IE aumenta, disminuye la percepción del impacto que las expectativas positivas de los profesores-tutores tienen sobre los alumnos: IE baja (85\%) media $(78 \%)$ y alta $(71 \%)$. Es este sentido, se confirma que las interacciones personales afectuosas y las expectativas elevadas resultan buenas prácticas vitales para conseguir escuelas de calidad (Beaudoin, 2008), por tanto, no sólo el alumno debe confiar en sus capacidades, sino que el profesor también debe creer en ellas (Furrer, 2013).
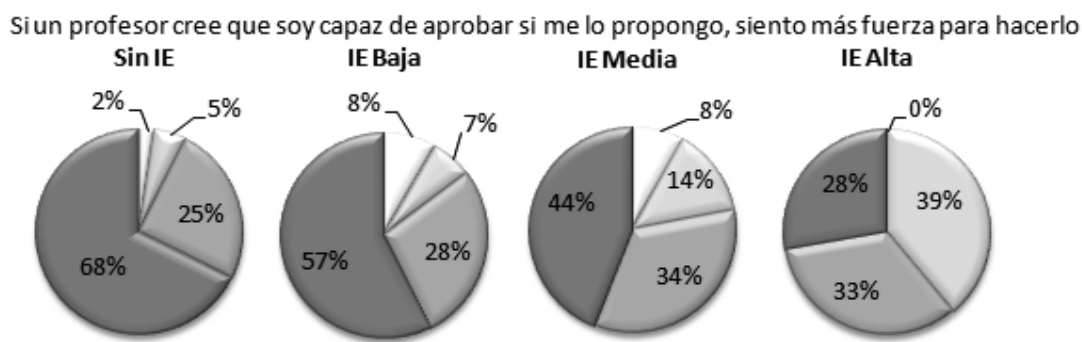

Creer en las posibilidades de un alumno afecta positivamente a la percepción que el alumno tiene de sí mismo

Tutores

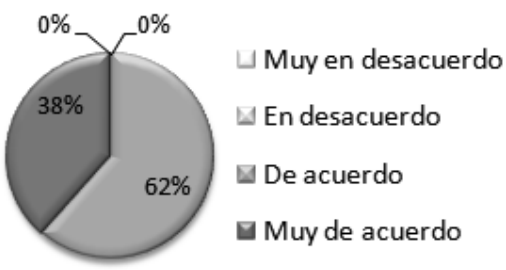

Figura 10. Visión positiva de las posibilidades de los alumnos

En cuanto a la imagen que se proyecta sobre el otro en relación a las normas y estándares sociales, se observa en la figura 11 que el 100\% de los profesores-tutores consideran que su comportamiento es para los alumnos un modelo adulto y por esa razón afirman que deben cuidarlo mucho. Los alumnos por su parte aportan respuestas dispares. Por un lado, los alumnos sin indicadores de IE afirman en un 59\% que los profesores cumplen las normas que ellos también deben cumplir. Los alumnos con un 
nivel bajo y medio de IE responden en el mismo sentido (42\% y $40 \%$ respectivamente), mientras que el alumnado con un nivel alto de IE percibe solo en un $22 \%$ de los casos que los profesores cumplen las normas tal y como se las hacen cumplir a ellos.

Se observa que la percepción de los alumnos sobre el grado de cumplimiento de las normas por parte de los profesores depende de la perspectiva de cada grupo de alumnos, siendo las diferencias importantes en función del grado de inadaptación escolar: a mayor inadaptación, la percepción sobre el profesorado es más negativa en el tema del cumplimiento de normas. Esto tiene, en la práctica, importantes repercusiones; tal y como plantea Pérez Pérez (2007), parece importante hacer un esfuerzo para clarificar tanto las normas como las consecuencias de su incumplimiento. Este hecho ha de tenerse en cuenta, ya que en la escuela también se forman la voluntad, la identidad personal, las emociones, las actitudes y los valores éticos (Ortega Esteban, 2005), lo que sin duda se ve mediatizado por la percepción por parte de los alumnos de si los profesores cumplen o no las normas en el espacio escolar. Beaudoin (2008: 121) sugiere al respecto lo que consideremos una buena práctica ineludible: "si queremos que los estudiantes sean éticos, debemos ejemplificarlo. Si queremos que los estudiantes sean respetuosos, debemos demostrarles cómo".

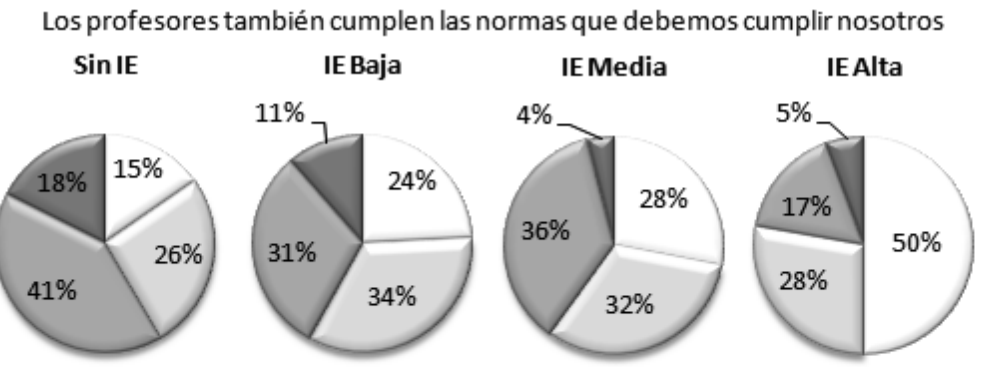

Micomportamiento es para los alumnos un modelo adulto, por esa razón debo cuidarlo mucho

Tutores

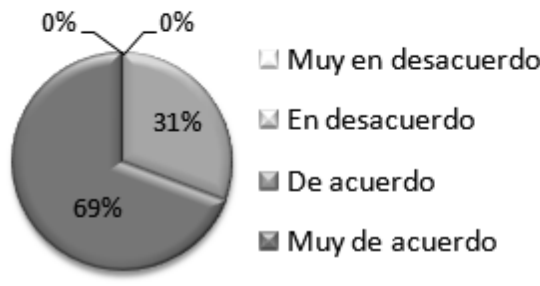

Figura 11. El profesor como modelo adulto

La figura 12 muestra que el $81 \%$ de los alumnos sin IE afirma que los profesores les ayudan a sentirse bien consigo mismos y con los demás, mientras que los alumnos con inadaptación escolar progresivamente se alejan de esa afirmación (IE baja 57\%, IE media 50\%, IE Alta 47\%), una tendencia que también se constató en otras respuestas relacionadas con la percepción social y emocional del profesorado por parte de los alumnos. Como señalan Santibáñez y Martínez-Pampliega (2013), el proceso educativo como proceso integral incluye no sólo los contenidos académicos. 


\section{Los profesores nos ayudan a sentirnos bien con nosotros mismos y con los demás}

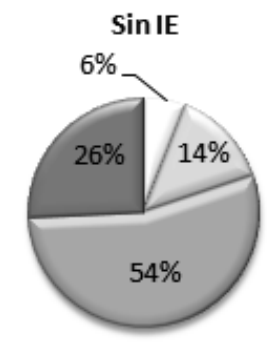

IE Baja

IEMedia
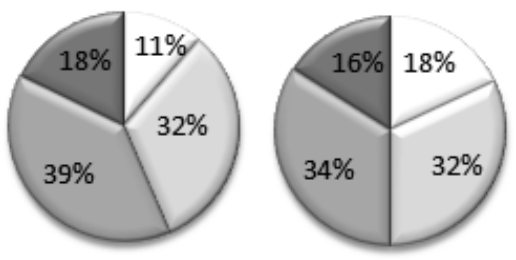

IEAlta

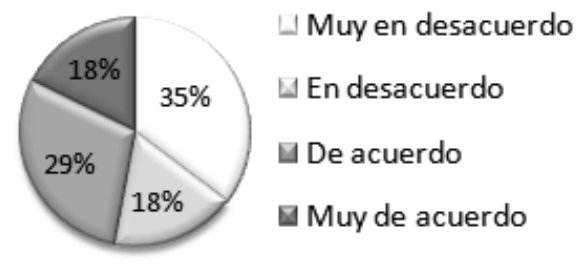

Figura 12. Labor socioeducativa

Por último, en la figura 13 se observan estrategias que orientan buenas prácticas docentes relacionadas con el desarrollo integral de los alumnos: el profesorado afirma masivamente, en un $92 \%$ de las respuestas, que evalúa el adecuado desarrollo académico, social y emocional de sus alumnos. Resulta congruente con lo afirmado por García García (2010) al afirmar que cuando se hace referencia a los aprendizajes, no sólo se trata de las materias y áreas disciplinares, sino también de procedimientos y estrategias, educación para la convivencia, para el desarrollo personal, cognitivo, afectivo, moral y social; así como los relacionados con el equilibrio emocional. Sin embargo, las respuestas de los profesores contrastan con la percepción que tienen los alumnos, de forma más acusada en el caso de alumnos con inadaptación escolar.

Evalúo el adecuado desarrollo académico, social y emocional de mis alumnos

Tutores

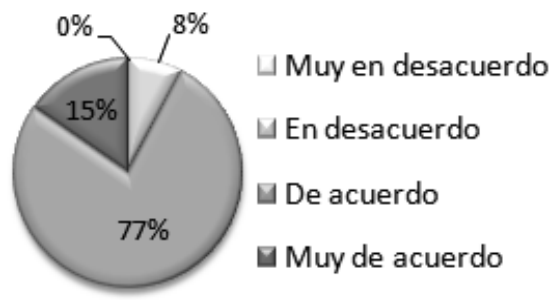

Figura 13. Estrategias de evaluación integral 


\section{Conclusiones}

Como conclusiones destacables del estudio realizado, se han identificado factores a tener en consideración en el desarrollo de buenas prácticas educativas con adolescentes vulnerables, relacionadas con el clima de aula:

- La importancia de una actitud de escucha activa por parte de los profesores.

- La gestión por parte del profesorado de las propias emociones, para utilizarlas como recurso educativo.

- La respuesta por parte de los profesores a la necesidad de los alumnos de un apoyo explícito y en público: necesitan sentirse valorados y apoyados.

- La necesidad de no crear estereotipos sobre los alumnos: éstos afectan también a la visión que los alumnos tienen de sí mismos.

- El cumplimiento por parte de los profesores de las normas destinadas a la comunidad educativa y que, por tanto, también deben cumplir los alumnos.

- Los profesores, como educadores, pueden promover la adquisición de habilidades sociales y de comportamiento de los alumnos.

Como se ha señalado, los resultados del presente trabajo muestran los importantes beneficios que suponen la actitud de escucha activa del profesorado hacia los alumnos y su disponibilidad para acompañarles en el proceso educativo. Ofrecer esas oportunidades de escucha y diálogo resulta fundamental, al igual que lo es para los alumnos encontrar un momento adecuado y un adulto dispuesto a escuchar; algo que no es fácil y resulta aún más complejo para los alumnos con inadaptación escolar. Como se ha visto, los alumnos con una mayor inadaptación escolar tienen una percepción menos favorable de las características socioemocionales del profesorado y se encuentran menos predispuestos a compartir cuestiones personales con sus profesores-tutores.

Por otra parte, la gestión de las emociones por parte del profesorado puede ser una importante oportunidad de crecimiento para los alumnos. Para ello la formación y, sobre todo, la predisposición para compartir esas emociones, son elementos fundamentales. En cualquier caso, son claramente percibidas por los alumnos y afectan de forma notable a la construcción del clima de aula.

Teniendo en cuenta, como se ha dicho anteriormente, que en la escuela se dan cita, entre otros factores, la interpretación de la realidad y la interacción personal, resulta congruente que el apoyo explícito de los profesores aparezca como un factor de mejora para los alumnos y que así sea percibido por ambos.

Sin embargo, resulta difícil que profesores y alumnos encuentren un nivel adecuado de comunicación, especialmente en el caso de los alumnos con mayor inadaptación escolar. Dificultad que aparece especialmente en el caso de la percepción del otro a través de terceros, que exige a los profesores mantenerse muy atentos para evitar la creación de estereotipos que afectarán, entre otras cuestiones, a la percepción que los alumnos tienen de sí mismos (Furrer, 2013), especialmente a los que presentan peores resultados académicos y comportamientos más inadaptados. En ambos casos parece que, en profesores-tutores y alumnos, influye la deseabilidad social al responder al cuestionario. Sin embargo, cuando a los alumnos se les pregunta directamente sobre sus percepciones, éstos se muestran mucho más sinceros que sus 
profesores: los alumnos que sienten una percepción negativa hacia ellos por parte de los profesores, consideran también que esos mismos profesores no consiguen de ellos una buena adaptación a la clase, que se sientan bien en clase. De alguna manera, si un alumno se ve percibido como problemático, se siente peor en clase y con más probabilidad de mostrar una conducta inadaptada; además, tal y como muestran los resultados, considera que una de las razones de su malestar radica en el profesor.

Cuando se valora el cumplimento de las normas de la comunidad educativa, es probable que los alumnos no siempre las cumplan porque se encuentran en un proceso educativo, incluso que las perciban como una imposición a veces injusta, tal y como se señala en el estudio. Esta percepción aumenta en los alumnos que presentan un cierto grado de inadaptación escolar. Pero en el aula son los profesores los encargados del cumplimiento de las normas, lo que les coloca en una posición de responsabilidad en el cumplimiento de esas mismas normas: son ejemplo adulto, modelo de comportamiento y transmisores de valores.

El clima de aula refleja también la interacción entre los profesores, los alumnos, la organización, el contenido objeto de enseñanza, las normas, los hábitos y los comportamientos, y condiciona en gran medida la adquisición de competencias académicas y enseñanza de contenidos. En este proceso, los profesores que promueven las habilidades sociales y de comportamiento de los alumnos, mejoran también la adquisición de competencias y contenidos.

La escuela puede además crear un espacio de acogida, prevención y ruptura de procesos de fracaso y exclusión para alumnos provenientes de contextos socio-familiares en riesgo: el sentido de pertenencia resulta fundamental para ellos, así como lo es para el ser humano, como individuo y como especie. Teniendo en cuenta además que si bien la inadaptación social no resulta determinante para el fracaso escolar, sí constituye un factor que se debe tener muy en cuenta y por tanto actuar en consecuencia desde las posibilidades que ofrece la escuela.

En consecuencia, la reflexión sobre buenas prácticas docentes con el objetivo de mejorar el clima de aula resultará fundamental para comprender la complejidad del proceso educativo, para lanzar una mirada que analice todos los elementos que lo configuran y que explican a su vez los procesos de éxito y fracaso escolar, así como de educación integral de los alumnos.

\section{REFERENCIAS BIBLIOGRÁFICAS}

Alvira, F. (2011). La encuesta: Una perspectiva general metodológica. Cuadernos metodológicos. Madrid: Centro de Investigaciones Sociológicas.

Amores, F. J., y Ritacco, M. (2011). Evaluar en Contextos de Exclusión Educativa. Buenas Prácticas e Inclusión Social. Revista Iberoamericana de Evaluación Educativa, 4(1), 90-108. Recuperado de http://www.rinace.net/riee/numeros/vol4-num1/art5.pdf.

Amores F. J., y Ritacco, M. (2012). Prácticas escolares ante la exclusión social. Estudio en la Educación Secundaria Obligatoria. Contextos Educativos, 15, 41-60. doi: $10.18172 /$ con.654.

Arellano, N. (2006). Las barreras en la comunicación no verbal entre docentealumno. Revista ORBIS, 4, 3-38. Recuperado de http://www.redalyc.org/articulo. oa?id=70920402. 
Arnaiz, P. (2011). Luchando contra la exclusión: buenas prácticas y éxito escolar. Innovación Educativa, 21, 23-35. Recuperado de http://hdl.handle.net/10347/6222.

Beaudoin, N. (2008). Una escuela para cada estudiante. La relación interpersonal, clave del proceso educativo. Madrid: Narcea.

Boyano, J. (2013). Convivencia escolar: el fortalecimiento de los lazos afectivos. Participación educativa, (2). Recuperado de http://ntic.educacion.es/cee/revista/n2/ n2art_jose_boyano.html.

Bueno, M. R., y Garrido, M. A. (2012). Relaciones interpersonales en educación. Madrid: Ediciones Pirámide.

Carbonero, M.A., Martín, L. J., Román, J. M., y Reoyo, N. (2010). Efecto de un programa de entrenamiento al profesorado en la motivación, clima de aula y estrategias de aprendizaje de su alumnado. Revista Iberoamericana de Psicología y Salud, 1(2), 117-138. Recuperado de http://www.redalyc.org/articulo.oa?id=245116406001.

Claro, J. (2013). Calidad en educación y clima escolar: apuntes generales. Revista Estudios Pedagógicos, 39(1), 347-359. Recuperado de http://mingaonline.uach. cl/scielo.php?script=sci_issuetoc\&pid=0718-070520130001\&lng=es\&nrm=iso.

Cornejo, R., y Redondo, J. (2001). El clima escolar percibido por alumnos de enseñanza media. Última Década, 15, 11-52. Recuperado de http://www.redalyc.org/ articulo.oa? id=19501501.

Comellas, M. J. (2013). El clima cotidiano en el aula. Contexto relacional de socialización, 3(3), 289-300. Recuperado de http://www.ejihpe.es/index.php/journal/ article/view/51.

Fryd, P. (Coord.) (2011). Acción socioeducativa con infancias y adolescencias. Miradas para su construcción. Barcelona: Editorial UOC.

Furrer, S. (2013). Comprendiendo la amenaza del estereotipo: definición, variables mediadoras y moderadoras, consecuencias y propuestas de intervención. Reidocrea, 2, 239-260. Recuperado de http://hdl.handle.net/10481/27787.

García Amilburu, M. (2002). La educación, actividad interpretativa. Madrid: Dykinson.

García García, E. (2010). Competencias éticas del profesor y calidad de la educación. Revista Electrónica Interuniversitaria del Profesorado, 13(4), 29-41. Recuperado de http://www.redalyc.org/articulo.oa?id=217015570003.

González Alemán, J. (2010). Análisis de diferentes intervenciones socioeducativas en educación social. EDU-PSICHO Revista Internacional de Investigación y Calidad Educativa y Psicológica, 2, 7-13. Recuperado de http://dialnet.unirioja.es/servlet/ articulo?codigo=3706849.

Hernández, L., y Hernández, C. (2011). Hacia un modelo dinámico y eficiente de formación del profesorado. Revista electrónica interuniversitaria de formación del profesorado, 14(1), 53-66. Recuperado de http://aufop.com/aufop/revistas/arta/ digital/158/1619.

Hernández-Guanir, P. (2009). Test autoevaluativo multifactorial de adaptación infantil (TAMAI) (6 ed.). Madrid: Tea.

Jiménez Morales, M., y López Zafra, E. (2013). Impacto de la Inteligencia Emocional Percibida, Actitudes Sociales y Expectativas del Profesor en el Rendimiento Académico. Electronic Journal of Research in Educational Psychology, 11(1), 75-98. Recuperado de http://www.redalyc.org/articulo.oa?id=293125761004. 
Lizasoain, L., y Angulo, A. (2014). Buenas prácticas de escuelas eficaces del País Vasco. Metodología y primeros resultados. Participación Educativa. 3(4), 17-27. Recuperado de http://ntic.educacion.es/cee/revista/pdfs/n4art_lizasoain_angulo.pdf.

López Gómez, E. (2013). Aproximación a la percepción y satisfacción del profesor tutor de Secundaria Obligatoria respecto a su labor. Revista de Investigación en Educación, 11(1), 77-96. Recuperado de http://webs.uvigo.es/reined/ojs/index. php/reined/article/view/602.

Lozano, A. (2003). Factores personales, familiares y académicos que afectan al fracaso escolar en Educación Secundaria. Revista Electrónica de Investigación Psicoeducativa y Psicopedagógica, 1(1), 43-66. Recuperado de http://hdl.handle.net/10835/726.

Melendro, M., González Olivares, A.L., y Rodríguez Bravo, A.E. (2013). Estrategias eficaces de intervención socioeducativa con adolescentes en riesgo social. Pedagogía Social. Revista Interuniversitaria, 22, 105-121.

Mena, L., Fernández Enguita y Riviére, M.J. (2010). Desenganchados de la educación: procesos, experiencias, motivaciones y estrategias del abandono y del fracaso escolar. Revista de Educación, (número extraordinario), 119-145. Recuperado de http://www.mecd.gob.es/revista-de-educacion/numeros-revista-educacion/ numeros-anteriores/2010/re2010/re2010_05.html.

Moreno Madrigal, C., Díaz Mujica, A., Cuevas Tamarín, C., Nova Olave, C. y Bravo Carrasco, I. (2011). Clima escolar en el aula y vínculo profesor-alumno: alcances, herramientas de evaluación, y programas de intervención. Revista Electrónica de Psicología Iztacala, 14(3), 70-84. Recuperado de http://www.iztacala.unam.mx/ carreras/psicologia/psiclin/principal.html.

Murillo Estepa, P., y Becerra Peña, S. (2009). Las percepciones del clima escolar por directivos, docentes y alumnado mediante el empleo de "redes semánticas naturales". Su importancia en la gestión de los centros educativos. Revista de Educación, 350, 375-399. Recuperado de http://dialnet.unirioja.es/servlet/articulo?codigo=3039321.

Ochoa Cervantes, A., y Peiró I Gregori, S. (2010). Estudio comparativo de las actuaciones de los profesores ante situaciones que alteran la convivencia escolar: el caso de Querétaro (México) y Alicante (España). Revista Electrónica Interuniversitaria de Formación del Profesorado, 13(4), 113-122. Recuperado de http://www. aufop.com/aufop/revistas/arta/digital/155/1611.

Ortega Esteban, J. (2005). La educación a lo largo de la vida: la educación social, la educación escolar, la educación continua... todas son educaciones formales. Revista de Educación, 338, 167-175. Recuperado de http://www.revistaeducacion. mec.es/re338_10.htm.

Pérez Pérez, C. (2007). Efectos de la aplicación de un programa de educación para la convivencia sobre el clima social de aula en un curso de $2^{\circ}$ de ESO. Revista de educación, 343, 503-529. Recuperado de http://www.mecd.gob.es/revista-de-educacion/ numeros-revista-educacion/numeros-anteriores/2007/re343/re343_21.html.

Santibáñez, R., y Martínez-Pampliega, A. (Coords.) (2013). Intervención comunitaria con adolescentes y familias en riesgo. Barcelona: Graó.

Simons, H. (2011). El estudio de caso: Teoría y práctica. Madrid: Morata.

Zárate, J. (2002). El arte de la relación Maestro Alumno. México: Instituto Politécnico Nacional. 